\title{
EFECTOS DE UN PROGRAMA \\ DE EDUCACIÓN FÍSICA \\ EN EL APRENDIZAJE DE ALUMNADO \\ UNIVERSITARIO CON DISCAPACIDAD \\ INTELECTUAL
}

\section{Effects of a Physical Education Program Developed through Service-Learning Methodology in Students' with Intellectual Disability}

\author{
Laura CAÑADAS \\ Universidad Autónoma de Madrid. ORCID. Facultad de Formación de Profesorado \\ y Educación. Departamento de Educación Física, Deporte y Motricidad Humana \\ laura.cannadas@uam.es \\ María Teresa CALle-Molina \\ Universidad Autónoma de Madrid. ORCID. Facultad de Formación de Profesorado \\ y Educación. Departamento de Educación Física, Deporte y Motricidad Humana
}

Recepción: 3 de abril de 2020

Aceptación definitiva: 8 de julio de 2020

Resumen: Esta investigación busca conocer qué aprendizajes de carácter declarativo ha adquirido el alumnado con discapacidad intelectual tras la participación en un programa de Educación Física a través de la metodología de Aprendizaje-Servicio y, además, valorar su percepción sobre el impacto que el desarrollo de este programa ha tenido en sus relaciones con otros estudiantes de la Facultad. Participaron 34 estudiantes con discapacidad intelectual que cursaban el Programa Promentor en el curso 2019/2020 en la Universidad Autónoma de Madrid. La información se recogió a través de diarios de aprendizaje del alumnado tras cada intervención y de asambleas al final de cada cuatrimestre. La información se analizó con el software MAXQDA v.2020. Los resultados muestran que los aprendizajes curriculares adquiridos propios del área estaban relacionados con los contenidos de salvamento acuático, hockey y habilidades gimnásticas y los aprendizajes transversales tenían que ver con el trabajo en equipo y el comportamiento cívico; los aprendizajes útiles para la vida diaria se identificaron con los contenidos de salvamento acuático y primeros auxilios, y, por último, se valoró muy positivamente la relación con el alumnado de grado en esta experiencia. 
Palabras clave: discapacidad intelectual; Educación Física; Aprendizaje-Servicio; inclusión.

AвsтRAст: The research aims to find out what learning of a declarative nature has been acquired by students with intellectual disabilities after participating in a Physical Education program through the Service-Learning methodology, and also to assess their perception of the impact that the development of this program has had on their relationships with other students in the Faculty. Thirty-four students with intellectual disabilities who were studying the Promentor Program in the 2019/2020 academic year at the Autonomous University of Madrid participated. The information was collected through students' learning journals after each intervention and through assemblies at the end of each four months. The information was analyzed with MAXQDA v.2020 software. The results show that the curricular learning acquired specific to this area was related to the contents of water rescue, hockey and gymnastic skills and the transversal learning was related to teamwork and civic behavior. Learning that was useful for daily life was identified with the content of water rescue and first aid and, finally, the relationship with the university students in this experience was valued very positively.

KeY words: intellectual disability; Physical Education; Service-Learning; inclusion.

\section{Introducción}

$\tau$

a Discapacidad Intelectual (DI), según la Asociación Americana de Discapacidades Intelectuales y del Desarrollo (AAIDD), es una limitación significativa tanto en el funcionamiento intelectual como en la conducta adaptativa (habilidades adaptativas conceptuales, sociales y prácticas) que aparece antes de los 18 años. Además, es preciso tener en cuenta la interacción dinámica y recíproca existente entre los agentes circunstanciales del individuo (AAIDD, 2011). La investigación ha mostrado que las personas con DI presentan mayores tasas de obesidad con respecto a la población general (Grondhuis y Aman, 2013) o una densidad mineral ósea menor que los grupos de referencia con tendencia a sufrir problemas de osteoporosis en la vida adulta (Geijer, Stanish, Draheim y Dengel, 2014). Estos, entre otros, son algunos de los múltiples factores que justifican la necesidad de generar planes de acción para el inicio de este colectivo en programas de actividad física (Hsieh, Rimmer y Heller, 2014; San Mauro et al., 2016).

Junto con los beneficios sobre la salud física, los programas de actividad física en esta población han mostrado otros beneficios como son: el incremento de su autonomía personal proporcionado por su bienestar físico (Vallbona, 2003); beneficios en los procesos de toma de decisiones (Abellán y Sáez, 2014); beneficios estéticos y actitudinales y aquellos que subyacen de estos (Moreno, 2005); beneficios en la gestión emocional y el desarrollo personal y social (Muñoz-Jiménez, Garrote-Rojas y Sánchez-Romero, 2017), e incluso un envejecimiento saludable (Alonso-Dasouto y Silva-Ganso, 2013), teniendo en cuenta que la esperanza de vida en general y de este colectivo en particular ha aumentado y que, por tanto, las cifras de personas con DI 
mayores de 65 años ha incrementado en los últimos años (Aguado, Alcedo, Arias y Rueda, 2007). Es por ello por lo que se hace necesaria la inclusión de este colectivo en programas sistemáticos de actividad física desde edades tempranas, propiciando hábitos que puedan extenderse durante toda la vida y que generen beneficios en la salud global del individuo.

Sin embargo, frecuentemente este colectivo queda excluido de programas de actividad física ofertados a la población en general y relegados a programas definidos menos numerosos por su especificidad después de la adolescencia. Por ello, en muchas ocasiones, estos programas de actividad física deben estar incluidos en la propia formación académica y profesional de la que participan estas personas, ya sea en centros educativos de escolarización obligatoria (Primaria, Secundaria, Educación Especial) o en programas formativos universitarios. Con este objetivo, algunas universidades españolas, en sus programas de formación laboral, han incorporado formación específica en actividad física y deporte.

En ocasiones, para el desarrollo de estos programas, se implementan propuestas formativas desde la metodología de Aprendizaje-Servicio (ApS). Esto, además de lo expuesto, permite acercar esta realidad al alumnado universitario en su formación inicial. Concretamente, el futuro profesorado que cursa el Grado en Maestro/a en Educación Primaria con mención en Educación Física (EF) y, además, alumnado que podría dedicarse a sectores deportivos que trabajen con una población adulta como es el que cursa el Grado en Ciencias de la Actividad Física y del Deporte (CCAFyD) (Sanz-Arribas, Calle-Molina, Aguado-Gómez y Garoz-Puerta, 2019; Santos-Pastor, Martínez-Muñoz y Cañadas, 2018, 2019; Ríos, 2003, 2010).

El ApS es una metodología basada en el aprendizaje experiencial, que combina el aprendizaje académico del alumnado con el servicio a la comunidad, todo ello acompañado de procesos explícitos de reflexión (Gelmon, Holland y Spring, 2018). Las dos partes que conforman la metodología (el aprendizaje y el servicio) deben estar integradas, obteniendo un beneficio mutuo. De esta manera, el alumnado universitario desarrolla competencias académicas, cívicas y personales mientras realiza un servicio social. Este servicio debe ser de calidad, con unos objetivos y actividades previamente definidos que influirán y mejorarán, por una parte, el propio contexto sobre el que se interviene y, por otra, el aprendizaje de los estudiantes en clase (Furco y Norvell, 2019). Por tanto, tan importante es que se garantice el aprendizaje del alumnado como la calidad del servicio ofrecido.

Sin embargo, habitualmente las investigaciones focalizan sus resultados atendiendo a la adquisición de competencias del alumnado universitario y olvidando los efectos que este tipo de intervenciones tienen sobre el servicio, las cuales están estrechamente ligadas a los resultados de su acción y a la calidad de su servicio. Así, podemos observar cómo son escasos los trabajos sobre actividad física y ApS que analizan el efecto del servicio en el colectivo receptor (Chiva-Bartoll et al., 2019) y, más concretamente, en población con discapacidad intelectual. Únicamente el estudio de SanzArribas et al. (2019) analiza el impacto de un programa de ApS en la adquisición de competencias para prevenir el accidente acuático por parte de población con DI, encontrando aprendizajes muy significativos tras la implementación del programa en 
la capacidad de este colectivo para resolver situaciones de ahogamiento de una persona en el medio acuático. Por otra parte, ninguna de las investigaciones que presentan resultados sobre intervenciones de ApS y actividad física con población con discapacidad intelectual recoge el impacto a nivel personal y social que dichos programas han podido suponer para el alumnado con DI.

Una vez justificada la pertinencia de este estudio y subrayando la importancia de conocer la calidad y el impacto del servicio prestado, esta investigación busca conocer qué aprendizajes de carácter declarativo ha adquirido el alumnado con discapacidad intelectual tras la participación en un programa de Educación Física a través de la metodología de ApS y, además, valorar su percepción sobre el impacto que el desarrollo de este programa ha tenido en sus relaciones con otros estudiantes de la Facultad.

\section{Metodología}

\subsection{Diseño}

Se ha llevado a cabo una investigación de corte cualitativo. Esta elección metodológica responde a la necesidad de conocer la realidad social educativa investigada y proponer mejoras concretas (Cerrón-Rojas, 2019). Este estudio tiene un diseño retrospectivo de naturaleza fenomenológica basado en la comprensión de la realidad a través del discurso de los participantes. Específicamente busca conocer los aprendizajes desarrollados por alumnado universitario con DI en un programa de Educación Física desarrollado con metodología $\mathrm{ApS}$ a través de la voz del propio alumnado.

\subsection{Programa de Educación Física}

El alumnado participante en esta investigación desarrolla su formación universitaria en el "Programa Promentor: Formación para la Inclusión Laboral" que se lleva a cabo en la Universidad Autónoma de Madrid (Cátedra de Patrocinio Universidad Autónoma de Madrid-Fundación Prodis). Este es un programa formativo cursos que busca dotar al alumnado con DI de competencias profesionales que faciliten su inclusión en el ámbito laboral (Izuzquiza y Rodríguez, 2015; Sanz-Arribas, Calle-Molina y Martínez-de-Haro, 2019).

Dentro de este programa, en cada uno de los cursos, se desarrolla una asignatura denominada "Desarrollo de conductas motrices y su relación con el ámbito deportivo" que tiene como objetivo mostrar diversas formas de realizar actividad física para aumentar su sentimiento de competencia, así como crear rutinas y hábitos físicos que deseen mantener al término del programa. En la estructura de esta asignatura, determinados contenidos se trabajan a través de la metodología de ApS. Para ello, estudiantes universitarios del Grado en Ciencias de la Actividad Física y el Deporte y el Grado en Maestro/a en Educación Primaria (Mención Educación Física) llevan a cabo, en algunas de las asignaturas que cursan, intervenciones sobre dichos contenidos con el objetivo de que el alumnado del Programa Promentor desarrolle determinados aprendizajes. 
Tiene como finalidad principal que el alumnado de los grados desarrolle las competencias profesionales relacionadas con la implementación de propuestas de actividad física y deporte mientras se cubren las necesidades del Programa Promentor en esta materia, aportando al alumnado con DI experiencias positivas en actividad físico-deportiva y aprendizajes relacionados con una ocupación activa del ocio y la adquisición de hábitos de práctica saludables (Santos-Pastor et al., 2018). Este proyecto se enmarcaría en la modalidad de servicio directo, en el cual el alumnado universitario participante trata de detectar una necesidad en la población con DI y lleva a cabo un plan de diseño, intervención y evaluación, buscando dar respuesta a esa necesidad.

\subsection{Participantes}

La selección de los participantes fue de forma incidental por estar cursando el Programa Promentor en el curso 2019/2020. En la investigación participaron un total de 34 estudiantes con DI, que suponen el $100 \%$ del alumnado matriculado en ese curso. La información completa de los participantes se encuentra en la Tabla 1 y la de las sesiones desarrolladas en la Tabla 2.

\begin{tabular}{|l|c|}
\hline \multicolumn{2}{|l|}{ TABLA 1. Relación de participantes en la investigación } \\
\hline Participantes \\
\hline Total, $n$ & 34 \\
\hline Curso, $n$ & 17 \\
\hline $1 .^{\circ}$ curso & 17 \\
\hline $2 .^{\circ}$ curso & \\
\hline Sexo, $n$ & 16 \\
\hline Mujeres & 18 \\
\hline Hombres
\end{tabular}

\begin{tabular}{|l|c|c|c|}
\hline \multicolumn{4}{|c|}{ TABLA 2. Sesiones desarrolladas } \\
\hline Sesiones & Total & $1 .^{\circ}$ curso & $2 .^{\circ}$ curso \\
\hline Total, $n$ & 17 & 6 & 11 \\
\hline Cuatrimestre, $n$ & 6 & 2 & 4 \\
\hline $1 .{ }^{\circ}$ cuatrimestre & 11 & 4 & 7 \\
\hline $2 .^{\circ}$ cuatrimestre & 2 & 0 & 2 \\
\hline Contenidos, $n$ & 4 & 2 & 2 \\
\hline Iniciación Deportiva & 3 & 0 & 3 \\
\hline Habilidades Gimnásticas & 6 & 3 & 3 \\
\hline Hockey & 2 & 2 & 0 \\
\hline Actividades Físico-Deportivas en el Medio Natural &
\end{tabular}

Ediciones Universidad de Salamanca / CC BY-NC-ND

Siglo Cero, vol. 51 (4), 2020, octubre-diciembre, pp. 53-68 


\subsection{Instrumentos}

Para la recogida de la información se emplearon los diarios de aprendizaje del alumnado y asambleas al final del cuatrimestre donde se les preguntaba por temas concretos de los aprendizajes desarrollados. Las preguntas incluidas en ambos instrumentos fueron diseñadas específicamente para esta investigación. El objetivo del diario era recabar información individual de cada uno de los participantes tras cada intervención, mientras que el objetivo de la asamblea era recoger información sobre el aprendizaje de todo lo trabajado en el cuatrimestre, pasado un tiempo desde que esos contenidos fueron desarrollados. Para la construcción de ambos instrumentos las investigadoras, partiendo de la experiencia previa de trabajo con este colectivo en cursos anteriores, se reunieron en diversas ocasiones. En primer lugar, para conceptualizar y acordar los aspectos que deberían formar parte de los instrumentos. En un segundo momento, para delimitar posibles preguntas a incluir en cada uno de estos instrumentos, adecuándolas a las capacidades y posibilidades del alumnado del título. Para finalizar, las investigadoras procedieron a reunirse para pulir matices de las preguntas planteadas, especialmente para facilitar la comprensión de las preguntas por parte del alumnado al que iban dirigidas.

Los instrumentos quedaron configurados de la siguiente forma: (a) en el diario de aprendizaje, tras cada sesión desarrollada por el alumnado universitario a través de la metodología de ApS debían contestar a la siguiente pregunta: Hoy has tenido clase con los chicos/as de Grado y nos gustaría que contestaras a una pregunta ¿Qué has aprendido en la clase de hoy sobre la actividad física que has realizado?; (b) al finalizar cada cuatrimestre (en enero y mayo) se realizaba una asamblea con todo el alumnado de forma conjunta. En ella se preguntaba sobre los siguientes temas: (a) ¿Qué has aprendido en las clases que has practicado estas semanas con el alumnado de Grado?; (b) ¿Creéis que algo de lo que habéis aprendido os va a servir para aplicarlo en vuestra vida diaria?; y (c) Que el alumnado de Grado haya sido quien ha dado las clases de los contenidos de Educación Física ¿te ha servido para mejorar tu relación con el resto de estudiantes de la Facultad? ¿Habéis notado la diferencia de antes de que estos chicos/as os diesen clase a después de habérosla dado? ¿En qué o cómo lo habéis notado? En las preguntas a y b de la asamblea, se iban valorando cada una de las áreas de actividad física trabajadas durante el cuatrimestre.

\subsection{Procedimiento}

Esta investigación se ha estructurado en 5 fases: (1) Organización del programa de EF: a finales del curso 2018/2019 se estableció un calendario de desarrollo de los diferentes contenidos de EF para el desarrollo del programa, estableciendo el número de sesiones máximas que podrían llegar a desarrollarse dentro de cada uno de los contenidos que se impartían; (2) Diseño del estudio: previo al comienzo del curso académico, conociendo cómo iba a ser la implementación de clases de EF en el Programa Promentor, se procedió al diseño de las fases que iban a guiar la recogida de información; (3) Elaboración de instrumentos: después se procedió a diseñar cada uno de los 
instrumentos; (4) Recogida de información: para la recogida de información de esta investigación, al alumnado de Promentor se le entregaba tras cada intervención un documento con la pregunta que debían responder. Este documento debía rellenarlo en el plazo de una semana e incluirlo junto con el resto de información en su diario de aprendizaje. Transcurrido este periodo de tiempo, se les recogía el documento. La asamblea, por su parte, se realizaba en enero, al finalizar el primer cuatrimestre, y en mayo, al finalizar el segundo cuatrimestre. Para ello, se reunía al alumnado de los dos cursos y las investigadoras acudían para realizar las preguntas previamente preparadas. Durante el desarrollo de la asamblea, dichas preguntas se concretaban y reconducían en función de las respuestas que daban los participantes. La información era grabada; (5) Análisis de la información: una vez finalizado el cuatrimestre, toda la información recogida era transcrita y se analizaba.

En esta investigación se ha garantizado el anonimato de las personas que han participado siguiendo las normas éticas de investigación nacional e internacional. Para cumplir con ello, se desligan las respuestas de los participantes de cualquier posibilidad de identificación, codificando las respuestas proporcionadas. Los datos resultantes del mismo se descargaron en un fichero Excel que se guardaba en un disco duro externo en el despacho de una de las profesoras responsables del estudio bajo llave. Los datos recabados eran exclusivamente empleados en este estudio.

\subsection{Análisis de la información}

Teniendo en cuenta el número de sesiones desarrolladas y el número de estudiantes, de las 289 posibles aportaciones en los diarios, únicamente 141 fueron respondidas y, por tanto, sometidas a análisis (71 en el primer cuatrimestre y 70 en el segundo; 71 aportaciones en el primer curso y 70 en el segundo curso). Las asambleas tuvieron una duración de 35 minutos en el primer cuatrimestre y 25 minutos en el segundo. Una vez recogida y transcrita la información de todos los instrumentos de este estudio, procedimos a analizarla. Para ello, se empleó el software de análisis de datos mixtos MAXQDA v.2020. Una lista de códigos se desarrolló de acuerdo con los tópicos de la investigación y la información obtenida tras la lectura detallada de los diarios y la transcripción de las asambleas, siguiendo el modelo de Taylor y Bogdan (1992). Este análisis fue desarrollado sobre una base cualitativa, codificando y categorizando la información. Después, la información se organizó a través de un sistema de codificación abierto, estableciendo los códigos en categorías y subtemas (Vaismoradi, Turunen y Bondas, 2013). Finalmente, la información se interpretó teniendo en cuenta el contexto en el que se ha recogido, las posibilidades de los participantes, etc.

Finalmente, encontramos 3 grandes categorías que atienden a los propósitos de esta investigación: (a) Aprendizajes curriculares; (b) Utilidad de los aprendizajes para la vida diaria; y (c) Relación con otros agentes de la Facultad. A su vez, la categoría de "Aprendizajes curriculares" se divide en: (a.1) Aprendizajes propios del área de Educación Física; (a.2) Aprendizajes transversales; y (a.3) Actividades desarrolladas. En el Gráfico 1 puede observarse la distribución final de códigos establecidos en la investigación. 


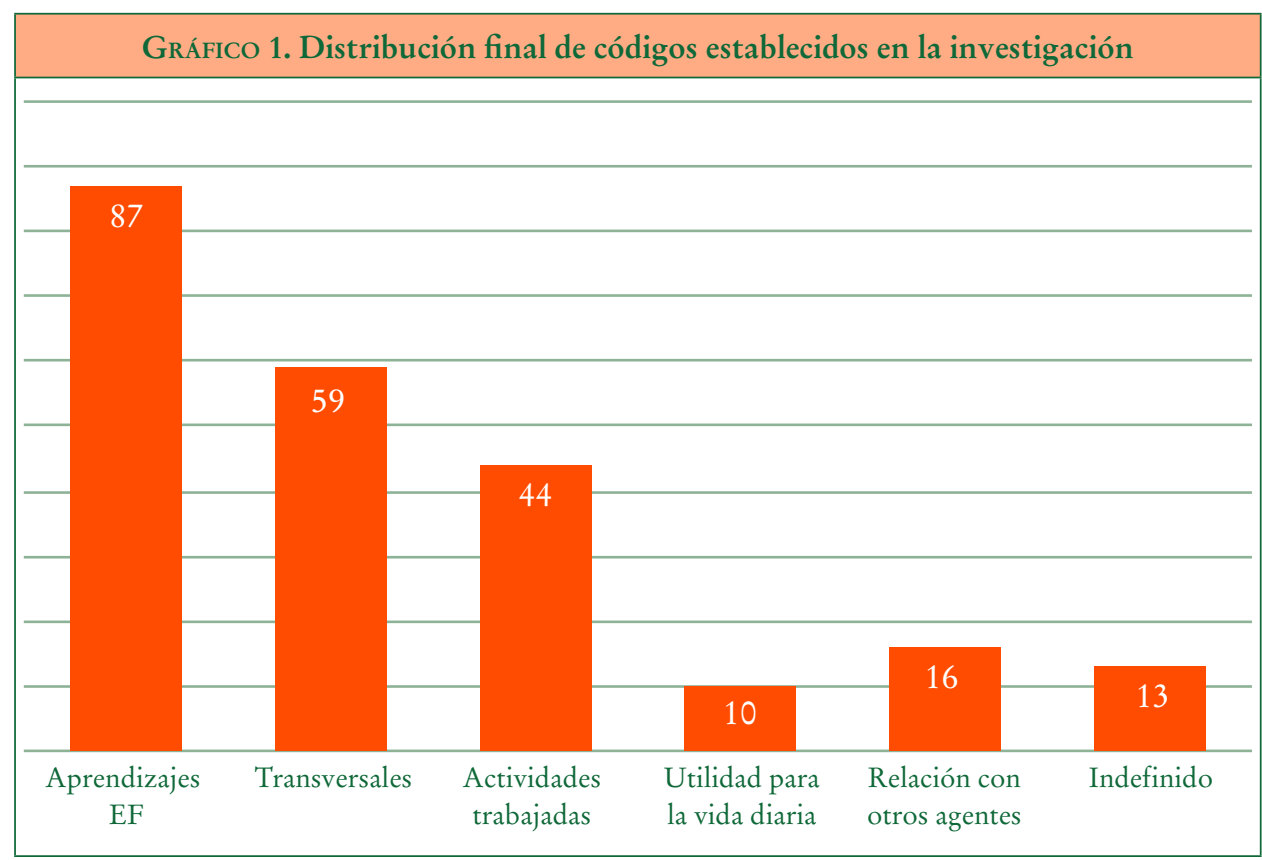

Para presentar la información esta ha procedido a ser codificada. En los diarios se presenta siguiendo el siguiente modelo: DIA_CURSO_PARTICIPANTE_FECHA. Por ejemplo: DIA_1_8_0304. Por su parte, la información recogida en la asamblea se presenta como: ASAM_CUATRIMESTRE-PARTICIPANTE. Por ejemplo: ASAM_1_8.

\section{Resultados}

Para la presentación de los resultados vamos a seguir la estructura presentada en el apartado de métodos y que responde a los instrumentos de recogida de información y las categorías extraídas tras el análisis de la información, quedando agrupada de la siguiente forma: (a) Aprendizajes curriculares; (b) Utilidad de los aprendizajes para la vida diaria; y (c) Relación con otros agentes de la Facultad.

\subsection{Aprendizajes curriculares}

En este apartado se analizará la información relativa a los aprendizajes curriculares desarrollados por el alumnado del Programa Promentor. A su vez, se divide en: (a.1) Aprendizajes propios del área de Educación Física; (a.2) Aprendizajes transversales; y (a.3) Actividades desarrolladas. 


\subsubsection{Aprendizajes propios del área de Educación Física}

En lo relativo a los aprendizajes propios del área de EF destacan varios de los contenidos trabajados por el alumnado. Así, en el primer curso destaca el aprendizaje sobre contenidos de salvamento acuático, con un $28 \%$ de las aportaciones en los aprendizajes propios del área de EF. Estas aportaciones se recogen de forma importante, tanto en diarios como en la asamblea:

[...] como hay que hacerlo es llamar primero al socorrista, luego coges el salvavidas, cuando estés en el bordillo tírate suave para ver a la persona, cuando la persona haya cogido el salvavidas pasa la mano por debajo, le pasas el salvavidas por encima de la persona, cógele de las manos, cuando ya lo tengas nada mirando a la persona hasta el bordillo (DIA_1_5_0304).

Lo que hemos aprendido en primeros auxilios es que cuando una persona se atragante le puede hacer una persona. Si una persona se atraganta, entonces hay que colocarle echado hacia delante, pero recto y hay que darle golpes secos en la espalda. Y si sigue mal hay que apretar un poco en el abdomen y llamar a urgencias (ASAM_2_10).

En el segundo curso, por su parte, se hace alusión sobre todo a los aprendizajes relativos al hockey y las habilidades gimnásticas, reconociendo aspectos básicos trabajados por el alumnado, como puede ser la forma de agarrar el stick de hockey o su conducción y los agarres básicos en el acrosport:

La mano dominante abajo y la mano no dominante arriba, y luego se conduce por la parte plana (ASAM_2_19).

Hemos aprendido en clase las reglas que son no levantar el stick al tirar a la portería y mirar a quién pasa girando el cuerpo hacia la persona. Hemos aprendido a conducir el stick con una pelota (DIA_2_28_0304).

He aprendido a confiar más en mis compañeros mediante agarres como: $1 .^{\circ}$ manomano; mano-antebrazo; antebrazo y de pinza (DIA_1_3_1912).

Otro era la mano entera, muñeca, antebrazo y luego también tenías que coger creo que era de la espalda, pero teniendo en cuenta que tenías que pisar los pies de tu compañero para tener equilibrio (ASAM_1_25).

\subsubsection{Aprendizajes transversales}

También se puede apreciar un gran número de referencias a aprendizajes transversales, especialmente aquello referido al trabajo en equipo. Aproximadamente, un $70 \%$ de las intervenciones hacen referencia al trabajo en equipo, destacando como el aprendizaje transversal más desarrollado.

He aprendido a comunicarme con los compañeros durante los juegos. También he aprendido a sentirme más participante con todos (DIA_2_22_2012). 
Trabajo en equipo. Organización de trabajo por equipo. Ayuda todos compañeros y compañeras (DIA_2_21_2012).

Otro tipo de aprendizajes transversales que se destacan, aunque en mucha menor medida, son los referidos a los comportamientos cívicos en las actividades en el medio natural: respeto al medio ambiente, no tirar desperdicios o respeto a la flora y fauna del lugar.

Hemos aprendido a recoger las cosas del suelo para que los pájaros no se lo coman, orientarse en la universidad (DIA_1_2_2205).

He aprendido que hay que ser un buen ciudadano y tirar las cosas a la basura (DIA_1_5_2205).

Y que si recogemos la basura en el parque los bosques quedan más bonitos y limpios y si encontramos basura, aunque no sea nuestra hay que recogerla (DIA_1_6_2205).

\subsubsection{Actividades desarrolladas}

Sin embargo, en muchas ocasiones, cuando se pregunta al alumnado por los aprendizajes desarrollados, se centran en hablar de las actividades desarrolladas y describirlas más que en explicar qué han aprendido con esas actividades. Esto destaca especialmente en el contenido de Actividades Físicas en el Medio Natural.

Hoy en las clases de EF hemos hecho una actividad dinámica por parejas dividida en tres partes. La primera parte consistía en encontrar una serie de letras en los pasillos de la UAM y coger una de cada punto, la segunda consistía en formar nombres de animales después de que nos dijeran una adivinanza. La tercera consistía en formar una palabra por pareja con los gestos y química entre compañeros (DIA_2_31_2404).

Diferentes juegos en el medio natural son unas actividades que teníamos que hacer. He aprendido el juego que trata nos dan un papel con el nombre del animal, hacer el ruido para que la otra persona que tenga el mismo animal y encontrarnos para hacer la pareja. He aprendido el juego de que uno de la pareja se tapa los ojos, la persona que está a tu lado te tiene que llevar hasta un objeto y dibujarlo en papel. He aprendido el juego se trata hacer por equipos y encontrar a un profesor que se escondía. Al acabar la clase hicimos una pequeña reflexión de lo que hemos hecho (DIA_1_8_0805).

También, en ocasiones, algunos estudiantes reportan este tipo de información en otros contenidos donde también han mostrado un dominio de los aprendizajes concretos del contenido, como puede ser el caso de salvamento acuático o las habilidades gimnásticas:

Lo primero que hemos hecho es una prueba de cómo salvar a la persona que se estaba ahogando. Luego hicimos unos largos de cada estilo. La segunda prueba teníamos que buscar pareja. Lo que teníamos que hacer una persona es la víctima, es decir, la persona que se ahoga y la otra persona es el que se salva (DIA_1_0304). 
He aprendido a jugar al Twister. He aprendido a jugar al escondite inglés con diferentes apoyos. He aprendido a intentar levantarme de espaldas, aunque no lo consiguiera. También he aprendido a levantarme con las manos. También el Twister consistía en que teníamos que ir andando y luego ir al aro y tocar con el pie o con las manos el aro (DIA_1_5_1212).

\subsection{Utilidad de los aprendizajes para la vida diaria}

Entre los elementos a investigar, también tenemos la utilidad de esos aprendizajes para la vida diaria. El alumnado destaca, de todos los contenidos trabajados, principalmente aquellos relacionados con el salvamento acuático y los primeros auxilios, y cómo esto puede servirles en situaciones donde otra persona esté en peligro de ahogamiento o haya sufrido un accidente.

Así podemos ayudar nosotros el día de mañana a alguien que esté en el agua (DIA_1_15_0304).

La RCP está bien saberlo porque nunca sabes cuándo te vas a encontrar con un accidente (ASAM_2_14).

Sin embargo, del resto de contenidos no hay muchas menciones a cómo creen que esto puede servirles o serles útil para utilizarlo en su tiempo libre, con sus amigos y/o familia o, incluso, en su futuro laboral.

\subsection{Relación con otros agentes de la Facultad}

El último apartado que surge en esta investigación es cómo el desarrollo de este programa a través de la metodología de ApS influye en su relación con otros agentes de la Facultad, principalmente el alumnado de los Grados de Maestro/a en Educación Primaria y CCAFyD. Sobre todo, destaca el conocer otros estudiantes de la Facultad que les ayudan en su formación y con los que pueden interactuar en su día a día.

Me han gustado mucho los juegos, los alumnos de grado son muy simpáticos y nos están ayudando y enseñando muchas cosas. Son muy divertidos y lo pasamos bien con ellos (DIA_1_0805).

Yo cuando voy por los pasillos siempre cuando hay alumnos de grado que ya me conocen también porque he ido de acampada, porque en grado y CCAFyD también son alumnos de aquí porque cuando yo paso por ahí me dicen, hombre, qué tal y yo bien (ASAM_2_28).

Yo también los veo en los pasillos, haciendo la clase y yo voy a saludar también y ellos también van a saludarme a mí, a preguntarme cómo estás (ASAM_1_15). 


\section{Discusión}

Los resultados de esta investigación muestran que el alumnado con DI reporta a nivel declarativo unos aprendizajes coherentes con el periodo de desarrollo del programa de Educación Física y los contenidos impartidos, además de valorar muy positivamente para su convivencia y socialización en la Facultad la impartición de esos contenidos por parte de alumnado universitario que desarrolla allí también su formación.

Nuestra investigación ha mostrado que el alumnado con DI puede aprender a nivel declarativo contenidos relacionados con la actividad física y deporte, a través de un programa de ApS, específicamente contenidos muy concretos como puede ser la forma de coger un stick de hockey y conducir la pelota, realizar un agarre en acrosport o conocer qué acciones deben llevar a cabo para salvar a una víctima de ahogamiento acuático. Esto coincide con investigaciones previas en las que obtienen resultados positivos en el aprendizaje de la población con DI para prevenir el accidente acuático, en este caso no solo a nivel declarativo, sino también a nivel procedimental (Sanz-Arribas et al., 2019).

Si nos centramos en programas de actividad física desarrollados con población con DI, estos se focalizan, por lo general, en la mejora de sus niveles de condición física o en la reducción de los niveles de obesidad, pero no en la creación de aprendizajes concretos sobre actividad física (Bertapelli, Pitetti, Agiovlasitis y Guerra-Junior, 2016). En contraste con nuestra investigación, centrarse únicamente en elementos de mejora de la salud física y con efecto a corto-medio plazo impedirá que la población con DI desarrolle su capacidad para practicar actividad física de forma autónoma en su tiempo libre.

En lo referente a la percepción del alumnado con DI sobre la mejora de sus relaciones y convivencia con los agentes implicados en el programa de Educación Física, no hemos encontrado estudios previos que hayan investigado esto específicamente. Otras investigaciones de ApS que desarrollan otro tipo de contenidos con población con DI también encuentran resultados positivos en las relaciones generadas entre los diversos agentes empleados, especialmente entre el alumnado y los receptores del servicio (Geva-López, 2016). Sin embargo, la mayoría de las investigaciones se centran en valorar este aspecto desde el punto de vista del alumnado universitario, donde se obtienen respuestas muy positivas respecto a su cambio de actitud hacia la población con DI y a las relaciones que establecen entre ellos (Gil-Gómez, Moliner-García, Chiva-Bartoll y García López, 2016; Woodruff y Sinelnikov, 2014), pero sin tener en cuenta qué supone esto para la población con DI en lo que a su convivencia habitual se refiere. Por otra parte, intervenciones de ApS en actividad física con otro tipo de población, por ejemplo, presos de una unidad psiquiátrica, también reflejan los beneficios que un programa de estas características tiene para esta población, permitiéndoles socializar entre ellos y con otros agentes sociales, además de mejorar conductas sociales (Lleixà y Ríos, 2013).

Entre las fortalezas que presenta este estudio encontramos que no existen apenas estudios de intervención con ApS que registren de forma concreta los resultados de 
la investigación sobre el colectivo receptor del servicio, especialmente cuando esto se refiere a población con DI. Además, se recoge el aprendizaje del alumnado en dos momentos diferentes, permitiéndonos contrastar si el aprendizaje declarado es cuestión de la inmediatez en el caso del diario, o si es un aprendizaje mantenido en el tiempo al ser compartido por el alumnado también en las asambleas. Por otra parte, este estudio también presenta ciertas limitaciones, como pueden ser las pocas intervenciones desarrolladas de cada uno de los contenidos, lo que limita los aprendizajes a unos muy concretos. Además, al ser la Educación Física una asignatura de marcado carácter procedimental, ese número pequeño de sesiones desarrolladas no permite poder valorar el desarrollo de los aprendizajes a ese nivel, suponiendo otra limitación de este trabajo.

Entre las futuras líneas de investigación, sería importante valorar el desarrollo de estos aprendizajes no solo a nivel declarativo, sino también a nivel procedimental observando y midiendo estos aprendizajes antes y después de la implementación del programa, y llevando un seguimiento de todo el alumnado durante los dos cursos que dura el título. También, sería interesante valorar la visión del alumnado universitario sobre el aprendizaje del alumnado con discapacidad intelectual y su cambio de perspectiva en la relación con este colectivo en su día a día en la Facultad tras su participación en este programa. Otro aspecto interesante sobre el que profundizar serían las emociones y sentimientos de la población con DI ante este tipo de propuestas.

\section{Conclusión}

Los resultados obtenidos en esta investigación indican que, por una parte, el alumnado con DI desarrolla aprendizajes propios del área de Educación Física cuando estos son impartidos por alumnado universitario que se está formando en el área. Además, el hecho de que esta formación sea desarrollada por alumnado universitario con el que comparten Facultad contribuye a mejorar sus relaciones con otros agentes con los que convive y comparte espacio de trabajo, ayudando a mejorar sus relaciones sociales en este contexto y a no relacionarse única y exclusivamente con sus compañeros y compañeras. Es importante que se lleven a cabo propuestas de estas características, ya que la etapa de formación educativa, en este caso universitaria, no debe ser única y exclusivamente un periodo de formación en el desarrollo de competencias dirigidas a desempeñar una labor profesional, sino también un periodo de desarrollo y crecimiento personal, y de desarrollo de competencias sociales y cívicas que permitan que ambos agentes, población con DI y alumnado universitario, se desenvuelvan en la sociedad con respeto y empatía hacia los otros.

\section{Agradecimientos}

En este apartado se quiere agradecer la inestimable colaboración del Programa Promentor de la Universidad Autónoma de Madrid para el desarrollo de esta investigación. 
EFECTOS DE UN PROGRAMA DE EDUCACIÓN FÍSICA EN EL APRENDIZAJE DE ALUMNADO

UNIVERSITARIO CON DISCAPACIDAD INTELECTUAL

LAURA CAÑADAS Y MARÍA TERESA CALLE-MOLINA

\section{Referencias bibliográficas}

AAIDD (2011). Discapacidad intelectual. Definición, clasificación y sistemas de apoyo social. Madrid: Alianza Editorial.

Abellán Hernández, J. y Sáez-Gallego, N. M. (2014). Justification of the motor test within the sport for people with intellectual disabilities. Revista Iberoamericana de Psicología del Ejercicio y el Deporte, 9(1), 143-153.

Aguado, A. L., Alcedo, M. A., Arias, B. y Rueda, M. B. (2007). Necesidades de las personas con discapacidad intelectual en proceso de envejecimiento. Bilbao: BFA DBF. Recuperado de http://www.bizkaia.net/Home2/Archivos/DPTO3/Temas/Pdf/topaketa16/Publicacion $\% 20$ Discapacidad \%20y\%20Envejecimiento.pdf.

Alonso-Dasouto, H. y Silva-Ganso, N. (2013). Exploración del efecto de la actividad física y su relación con distintas variables relevantes para un envejecimiento saludable en personas mayores con discapacidad intelectual. International Journal of Developmental and Educational Psychology, 1(2), 165-173.

Bertapelli, F., Pitetti, K., Agiovlasitis, S. y Guerra-Junior, G. (2016). Overweight and obesity in children and adolescents with Down syndrome-prevalence, determinants, consequences, and interventions: A literature review. Research in Developmental Disabilities, 57, 181-192. http://dx.doi.org/10.1016/j.ridd.2016.06.018

Campo, L. (2014). Aprendizaje servicio y educación superior. Una rúbrica para evaluar la calidad de proyectos. Tesis doctoral. Universitat de Barcelona. Recuperado de https://www. uv.es/aps/doc/Tesis/Tesis\%20Campo\%20Cano,\%20Laura.pdf.

Chiva-Bartoll, O., Ruiz-Montero, P. J., Martín-Moya, R., Pérez-López, I., Giles-Girela, J., García-Suárez, J. y Rivera-García, E. (2019). University Service-Learning in Physical Education and Sport Sciences: a systematic review. Revista Complutense de Educación, 30(4), 1147-1164. https://doi.org/10.5209/rced.60191

Furco, A. y Norvell, K. (2019). What is service-learning? Making sense of the pedagogy and practice. En P. Aramburuzabala, L. McIlrath y H. Opazo (Coords.), Embedding Service-Learning in European Higher Education. Developing a culture of civic engagement (pp. 13-36). Nueva York: Routledge.

Geijer, J., Stanish, H., Draheim, C. y Dengel, D. (2014). Bone mineral density in adults with Down syndrome, intellectual disability, and nondisabled adults. American Journal on Intellectual and Developmental Disabilities, 119(2), 107-114. https://doi.org/10.1352/19447558-119.2.107

Gelmon, S. B., Holland, B. A. y Spring, A. (2018). Assessing service-learning and civic engagement. Principles and techniques. Boston: Campus Compact.

Geva-López, E. (2016). La formación ética y en valores en la universidad y su relación con la calidad de vida de las personas con discapacidad intelectual. Una experiencia de aprendizaje-servicio. Tesis doctoral inédita. Barcelona: Universidad de Barcelona.

Gil-Gómez, J., Moliner-García, O., Chiva-Bartoll, Ó. y García López, R. (2016). Una experiencia de aprendizaje-servicio en futuros docentes: desarrollo de la competencia social y ciudadana. Revista Complutense de Educación, 27(1), 53-73. http://dx.doi.org/10.5209/ rev_RCED.2016.v27.n1.45071

Grondhuis, S. N. y Aman, M. G. (2013). Overweight and obesity in youth with developmental disabilities: a call to action. Journal of Intellectual Disabilities Research, 58, 787-799. https://doi.org/10.1111/jir.12090

Ediciones Universidad de Salamanca / CC BY-NC-ND

Siglo Cero, vol. 51 (4), 2020, octubre-diciembre, pp. 53-68

$$
-66-
$$


Hsieh, K., Rimmer, J. H. y Heller, T. (2014). Obesity and associated factors in adults with intellectual disability. Journal of Intellectual Disabilities Research, 58, 851-863. https://doi. org/10.1111/jir.12100

Izuzquiza, D. y Rodríguez, P. (2015). Un análisis de resultados de la primera experiencia de formación en España para personas con discapacidad intelectual en el ámbito universitario. Sindrome de Down. Vida Adulta Revista, 19, 1-23.

Lleixà, T. y Ríos, M. (2015). Service-Learning in Physical Education Teacher Training. Physical Education in the Modelo Prison, Barcelona. Qualitative Research in Education, 4(2), 106-133. http://dx.doi.org/10.17583/qre.2015.1138

Moreno, A. (2005). Incidencia de la actividad física en el adulto mayor. Revista Internacional de Medicina y Ciencias de la Actividad Física y el Deporte, 5(19), 222-237. Recuperado de http://cdeporte.rediris.es/revista/revista20/artvejez16.htm.

Muñoz-Jiménez, E. M., Garrote-Rojas, D. y Sánchez-Romero, C. (2017). La práctica deportiva en personas con discapacidad: motivación personal, inclusión y salud. Revista INFAD de Psicología. International Journal of Developmental and Educational Psychology, 4(1), 145-152. https://doi.org/10.17060/ijodaep.2017.n1.v4.1037

Ríos, M. (2003). El juego y el alumnado con discapacidad intelectual y/o plurideficiencia. Tándem: Didáctica de la Educación Física, 4(11), 40-49.

Ríos, M. (2010). La Educación Física en la Educación Social: una experiencia de aprendizaje y servicio. En M. MARTínez, Aprendizaje servicio y responsabilidad social de las universidades (pp. 113-128). España: Octaedro.

San Mauro, I., García de Angulo, B., Onrubia, J., Pina, D., Fortúnez, E., Villacorta, P., ... y Garicano Vilar, E. (2016). Nutrición y actividad física en personas con discapacidad intelectual. Revista Chilena de Nutrición, 43(3), 263-270. http://dx.doi.org/10.4067/ S0717-75182016000300005

Santos-Pastor, M. L., Cañadas, L., Martínez-Muñoz, L. F., Calle-Molina, M. T., Garoz-Puerta, I., Sanz-Arribas, I., Álvarez-Barrio, M. J. y Ponce-Garzarán, A. (2018). Programa de Educación Física para jóvenes universitarios con discapacidad intelectual desde la Metodología de Aprendizaje-Servicio. En F. J Murillo (Coord.), Avances en democracia y liderazgo distribuido en educación: Actas del II Congreso Internacional de Liderazgo y Mejora de la Educación (pp. 136-139). Madrid: RILME.

Santos-Pastor, M. L., Martínez-Muñoz, L. F. y Cañadas L. (2018). Actividades físicas en el medio natural, aprendizaje-servicio y discapacidad intelectual. Espiral. Cuadernos del Profesorado, 11(22), 52-60. http://dx.doi.org/10.25115/ecp.v11i22.1917

Santos-Pastor, M. L., Martínez-Muñoz, L. F. y Cañadas, L. (2019). La evaluación formativa en el aprendizaje-servicio. Una experiencia en actividades físicas en el medio natural. Revista de Innovación y Buenas Prácticas Docentes, 8(1), 110-118.

Sanz-Arribas, I., Calle-Molina, M. T., Aguado-Gómez, R. y Garoz-Puerta, I. (2019). Adquisición de competencias para prevenir el accidente acuático: un proyecto ApS con estudiantes universitarios y población con diversidad funcional. Publicaciones, 49(4), 219239. 10.30827/publicaciones.v49i4.11737.

Sanz-Arribas, I., Calle-Molina, M. T. y Martínez-De-Haro, V. (2019). Efectos de una formación inclusiva para la prevención del ahogamiento en personas con discapacidad intelectual. Retos, 35, 289-293.

TAYLOR, S. y Bogdan, R. (1992). Introducción a los métodos cualitativos de investigación. Barcelona: Paidós. 
Vaismoradi, M., Turunen, H. y Bondas, T. (2013). Content analysis and thematic analysis: Implications for conduction a qualitative descriptive study. Nursisng and Health Sciences, 15, 398-405. https://doi.org/10.1111/nhs.12048

VAllbona, C. (2003). La actividad física como elemento de salud para personas discapacitadas. En J. O. Martínez Ferrer (Ed.), Conferencia Internacional sobre Deporte Adaptado. Libro de Actas (pp. 283-296). Málaga: Instituto Andaluz del Deporte.

Woodruff, E. A. y Sinelnikov, O. A. (2014). Teaching young adults with disabilities through service learning. European Physical Education Review, 21(3), 292-308. https://doi. org/10.1177/1356336X14564171

Ediciones Universidad de Salamanca / CC BY-NC-ND

Siglo Cero, vol. 51 (4), 2020, octubre-diciembre, pp. 53-68

$$
-68-
$$

